\title{
Reconfiguration Modeling of Reconfigurable Hybrid FSO/RF Links
}

\author{
Hassan Moradi, Maryam Falahpour, Hazem H. Refai \\ Electrical and Computer Engineering \\ University of Oklahoma \\ Tulsa, OK, USA \\ \{hmoradi,maryam,hazem\}@ou.edu
}

\author{
Peter G. LoPresti \\ Electrical Engineering \\ University of Tulsa \\ Tulsa, OK, USA \\ pgl@ohm.ee.utulsa.edu
}

\author{
Mohammed Atiquzzaman \\ Computer Science \\ University of Oklahoma \\ Norman, OK, USA \\ atiq@ou.edu
}

\begin{abstract}
The purpose of devising a hybrid FSO/RF (Free Space Optics/Radio Frequency) system is to allow for reconfiguration, which enables an increased availability and reliability grade of the communication system. In the event of loss of an active FSO link, path reconfiguration ensures utilization of the FSO path queued next in priority. Thus, communication can be maintained in the optical domain with increased efficiency for a longer period. However, for all its benefits, path reconfiguration may inadvertently cause increased system delay and lower throughput, as well as packet loss. Given these advantages and disadvantages, path reconfiguration studies for hybrid systems are of great importance. A mathematical investigation and statistical consideration of path reconfiguration probability is the focus of this theoretical paper. A closed-form expression of the reconfiguration probability will be derived. The first-order Markov chain will provide a mathematically tractable model for atmosphere turbulence fading channels, as it uses only the received SNR of the FSO symbol immediately preceding the current one. Results show that increasing the intensity of turbulence-induced lognormal fading for FSO link will not necessarily increase the reconfiguration probability.
\end{abstract}

Keywords- Reconfiguration, Markov model, FSMC, FSO channel, Radio Frequency (RF), lognormal fading, and reconfiguration probability.

\section{INTRODUCTION}

Reinstating a failed connection in an optical network, thus improving system reliability, is the objective of path reconfiguration in a hybrid Free Space Optics/Radio Frequency (FSO/RF) [1]. This is accomplished by rerouting traffic either via an alternate optical link or to an RF link when an optical connection using transceivers is unavailable. Therefore, a connection is always established. For this reason, we use term reconfiguration probability instead of outage probability in reconfigurable Hybrid FSO/RF systems. In Hybrid FSO/RF systems, reconfiguration means rerouting the traffic from one path to another. The new path can be built of FSO links, RF links or a combination of them.

Packet loss occurs during reconfiguration at a point in time when packets should be retransmitted after a new connection is established. While a topology control mechanism can combine algorithms for topology reconfiguration, this subject is outside the scope of this paper. The objective of this paper is to determine how mathematically to characterize the reconfiguration statistics in a Hybrid FSO/RF network. However, we just focus on the statistics of FSO links.
The Finite State Markov Channel model (FSMC) representation [2], which originated as an extension of an earlier, less complicated model known as the Gilbert-Elliot channel [3] [4] is used. It helps to understand and characterize the probability issues. In the FSMC model, the fading process is related to the received SNR as a representation of channel quality [5]. An analytical method in terms of propagation attenuation and practical statistical measurements can be found in [6], while [7] takes into account the availability explanations as a function of link range and link budgets, as well as visibilitylimiting weather conditions. A second or higher-order Markov process should provide a more accurate model, although achieved through a higher degree of complexity. Wang and Chang [8] verified the first-order Markovian assumption between channel symbols in a Rayleigh fading environment.

The functionality of the first-order Markovian assumption is mathematically confirmed by showing that given the information corresponding to the previous symbol, the amount of uncertainty remaining in the current symbol should be negligible. There are two previous works of using Markov property for modeling FSO communication systems [9]. The first one investigates angular distribution of laser in a multiple scattering medium. However, as mentioned before, the argument is not useful for our analysis. Our work does not overlap the aforementioned work, but rather characterizes the reconfiguration (i.e. rerouting) probabilities in a hybrid $\mathrm{FSO} / \mathrm{RF}$ network that is suffering scintillation turbulence in its optical links.

The reminder of this paper is organized as follows: Section II gives the system and SNR model for OOK modulation. Section III presents the Markov application for fading channels along with the FSMC model. Section IV provides an analytical discussion of reconfiguration probabilities for lognormal fading channel, including stationary and transition probabilities, and Section $\mathrm{V}$ provides a conclusion to the paper.

\section{SNR MODEL}

On-off keying (OOK) modulation is often used to transmit optical information bits. The received signal $i_{d}(t)$ by OOK modulation can be expressed by

$$
i_{d}(t)=h(t) s(t)+i_{n}(t)
$$

where $s(t) \in\{0,1\}$ is the transmitted signal; $h(t)$ is the normalized channel fading intensity due to atmospheric turbulence and considered to be constant over a large number of transmitted 
bits; and $i_{n}(t)$ is total additive noise. For simplicity, investigators neglect term time $(t)$ in the analysis presented herein. The averaged received SNR for a Gaussian channel (no fading, $h=1$ ) can be given by [10]

$$
\gamma_{G}=\frac{4 R^{2} P_{t}^{2}}{\left(\sigma_{1}+\sigma_{0}\right)^{2}}
$$

where $\sigma_{1}$ and $\sigma_{0}$ are the standard deviation values of the noise currents for bits ' 1 ' and ' 0 ', respectively, and where $\operatorname{erfc}($.$) is the$ complementary error function. In Eq. (2) $R$ is the receiver's responsivity and $P_{t}$ is the average of transmitted power when no attenuation is assumed in the transmission model. For compatibility with (1), we can assume $2 P_{t} R=1$. More details about noise parameters can be found in [10]. For an FSO channel with a fading channel coefficient $h$ and additive Gaussian noise, the instantaneous SNR will be

$$
\gamma=\frac{4 h^{2} R^{2} P_{t}^{2}}{\left(\sigma_{1}+\sigma_{0}\right)^{2}}
$$

However, the averaged received SNR for a Lognormal channel can be defined the same as Eq. (2) by

$$
\bar{\gamma} \triangleq \frac{4 R^{2} P_{t}^{2}}{\left(\sigma_{1}+\sigma_{0}\right)^{2}}
$$

\section{MARKOV MODELING OF FADING CHANNELS}

A 2008 published study [11] provides modeling for the finite-state fading channels by the FSMC Markovian model. Sadeghi et al. historically integrated and reviewed works using the Markov chain for characterizing a finite-state fading channel. The paper describes properties for theoretical analysis of channel probabilities through fading. Two main approaches for the modeling of discrete Rayleigh fading channels are introduced in [5], namely the FSMC and the hidden Markov models (HMM). Both are based on the 1995 work by Wang and Moayeri [2], widely viewed to have revived FSMC modeling of Rayleigh fading channels in mobile radio communications. This work [2] emerged as the generally accepted FSMC case study from the earlier work by Gilbert [3] and Elliott [4] at Bell Labs. Since FSMC is a useful method for modeling fading channels, these principals in particular were used for theoretical modeling of the reconfiguration process through an optical link.

\section{A. FSMC Principles}

When discussing non-deterministic systems, it is assumed that $X_{n}, n \geq 0$, are random variables defined on a common property space, where $n$ is the time index [12]. Many examples exhibit a similarly important property, namely that the future evolution of the system (value of $X_{n+1}$ ) can be predicted having only the present state of the system (the value of $X_{n}$ ), i.e., history is irrelevant. Mathematically, this is represented by

$$
\begin{gathered}
P_{r}\left(X_{n+1}=y \mid X_{0}=x_{0}, X_{1}=x_{1}, \ldots, X_{x}=x_{n}\right) \\
=P_{r}\left(X_{n+1}=y \mid X_{n}=x_{n}\right)
\end{gathered}
$$

This is referred to as the Markov property, where $X_{n}$ is the discrete-time continuous Markov chain and $x_{0}, \ldots, x_{n}$ and $y$ are called the states. Many significant examples of random process assume a finite number of values $(\{0,1, \ldots, n\})$ or an infinite number of values $(\{0,1, \ldots, n, \ldots\})$. It is important to note that FSMC does not represent a Markov chain with finite $n$, but rather with finite $x_{n}$. Hence, for a $K$-state FSMC process, we have $n=0,1,2, \ldots$ and $x_{n} \in\{0,1,2, \ldots, K-1\}$. A FSMC is uniquely defined by three components: states, transition probability matrix, and stationary state probability vector.

After state, the stochastic component of the $K$-state FSMC model is the state transition probability, i.e., the probability of moving from state $x$ to state $y$. It is denoted by [12] that:

$$
p_{x y} \triangleq P_{r}\left(X_{n}=y \mid X_{n-1}=x\right)
$$

The transition probabilities in turn give rise to a $K \times K$ transition matrix $\bar{P}$. It is obvious, for example, a two-state stochastic process with $x, y \in\{0,1\}$ can be defined as

$$
p_{10}=P_{r}\left(X_{n}=0 \mid X_{n-1}=1\right)
$$

The second stochastic component of the FSMC model is the stationary (or steady state) probability vector, represented by the probability of being in state $k$ at any time index $n$ :

$$
\pi_{n}(k)=P_{r}\left(X_{n}=k\right)
$$

Important characteristics of the FSMC components are summarized in [2], while details about the Markov chains can be found in [12]. The aforementioned definition for a finite state Markov chain is considered as a first-order FSMC. A first-order Markovian assumption in the FSMC model is defined as the previous fading channel state where the current state is statistically independent of all other past and future fading channel states. By contrast, in higher-order Markov models, the probability of transition to a state depends on previous states. Details about second order Markov chains can be found in [12, chap. 4].

\section{B. FSMC Model for Optical Reconfiguration}

Sadeghi et al. [11] claim the first-order FSMC models are more accurate for slow fading channels than for medium-rate or fast-fading channels. In the channel model, each state corresponds to a specific channel quality. The Channel State Information (CSI) is usually determined by channel estimation and may be available through analysis. The following is a discussion of the reconfiguration process in terms of statistical probability.

Our FSMC model can be defined as a three-state Markov chain at any time $n$

$$
X_{n}= \begin{cases}S_{0} & \text { noreconfiguration is required at timen } \\ S_{1} & \text { hysteresis gap } \\ S_{2} & \text { reconfiguration is required at timen }\end{cases}
$$

Fig. 2 shows the state diagram represented by the chain of three states. The transition probability matrix is defined as

$$
\bar{P}=\left[\begin{array}{lll}
p_{00} & p_{01} & p_{02} \\
p_{10} & p_{11} & p_{12} \\
p_{20} & p_{21} & p_{22}
\end{array}\right]
$$

In-state transition probabilities, $p_{i i}$ and the adjacent state transition probabilities $p_{i j}(i \neq j)$ are shown in Fig. 2(b), where $i$ and $j$ represent the states and can be 0,1 or 2 .

The reconfiguration mechanism uses two thresholds from the received signal strength of the optical wireless link. One threshold level called-reconfiguration threshold -is selected based on the minimum required SNR by the receiver. The other-switch back threshold - is used to restart transmission on the old optical wireless link. From this, it can be supposed that 
reconfiguration will occur when the received $\mathrm{SNR}, \gamma$ is less than the reconfiguration threshold value $\gamma_{L}$. Reconfiguration occurs at time $t_{0}$ in Fig. 2(a), while switch back to the original configuration begins once the received SNR improves to $\gamma_{H}$ at time $t_{1}$.

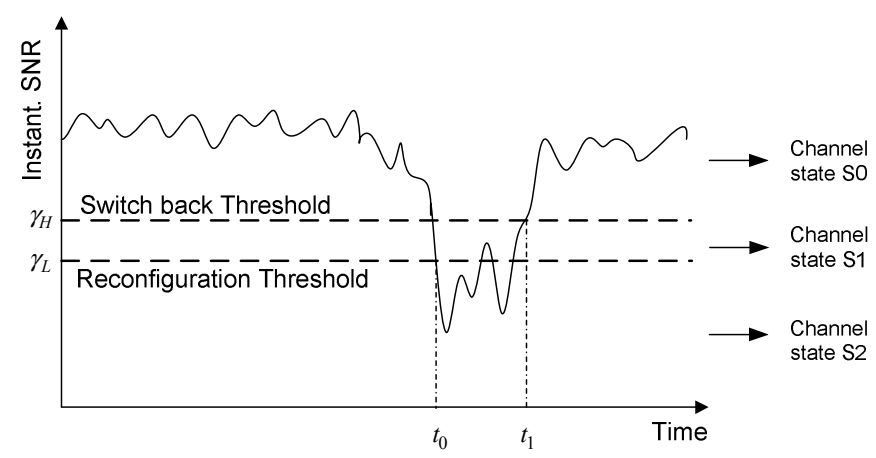

(a)

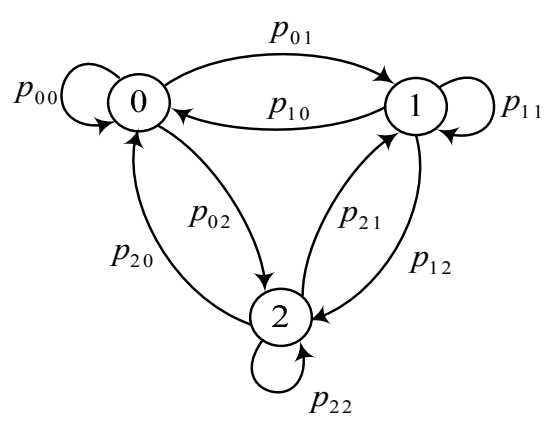

(b)

Fig. 2: (a) An example of the relationship between received SNR of the fading channel and reconfiguration times. (b) State transition diagram.

It is clear that just two parameters $p_{12}$ and $p_{10}$ denote to the reconfiguration probabilities:

$p_{12}$ : the probability that an FSO link is going to be obscured and needs to be reconfigured. The traffic can be directed to a different FSO link or even an RF link as backup. We call this parameter as reconfiguration probability.

$p_{10}$ : the probability that the link can be recovered from an obscured state. We call this parameter as switch back probability.

In addition, the stationary probabilities can be defined as:

$\pi_{n}(0)$ : the probability that an FSO link be in state 0 , which means it is in an active condition.

$\pi_{n}(2)$ : the probability that the link be in state 2 , which means it is obscured.

$\pi_{n}(1)$ : the probability that the link be in state 1 ; the status of the link depends on its previous state.

\section{RECONFIGURATION PROBABILITIES IN LOGNORMAL FADING}

In the presence of atmospheric turbulence, i.e., scintillation fading with scintillation index S.I. as lognormal distribution, the BER will be given by

$$
P_{e}^{L}=\frac{1}{2} \int_{-\infty}^{\infty} f_{x}(X) \operatorname{erfc}\left(\frac{\sqrt{2} R P_{t} e^{2 X}}{\left(\sigma_{1}+\sigma_{0}\right)}\right) d X
$$

where $f_{x}(X)$ is the probability density function of normally distributed variable $x$ with mean $\mu_{x}$ and variance $\sigma_{x}^{2}$, with

$$
\sigma_{x}=\frac{\sqrt{\ln (S . I .+1)}}{2}
$$

In scintillation fading, we can make the average power loss due to atmospheric fading unity; fading does not, on average, attenuate or amplify the optical power. The authors choose $\mu_{x}=-\sigma_{x}^{2}$, where the fading coefficient is usually normalize as $E[h]=1$ [13]. Thus, the lognormal distribution will be defined by

$$
f_{x}(h)=\frac{1}{\sqrt{8 \pi} h \sigma_{x}} \exp \left(-\frac{\left[\ln (h)+2 \sigma_{x}^{2}\right]^{2}}{8 \sigma_{x}^{2}}\right)
$$

where $h$ is the magnitude of fading coefficient, which is the ratio of the faded light intensity to the intensity without turbulence $h=I_{n} / I_{0}$.

\section{A. State Probabilities}

We assume that noise statistics (i.e. variances) are constant over the observation period. Also, we assume that the fluctuation due to channel fading is very small when compared to bit interval. These assumptions enable us to discuss the reconfiguration analysis on fluctuation of the fading coefficient. Recalling a lognormal distribution for normalized irradiance $h$ by (13), the probability of being a link at state 2 at time $n$, which means it is either obscured or too noisy,is expressed in terms of stationary probabilities, and can be rewritten using (3) by

$$
\pi_{n}(2)=\int_{0}^{\frac{\left(\sigma_{1}+\sigma_{0}\right) \sqrt{\gamma_{L}}}{2 R P_{t}}} f_{x}(h) d h
$$

Substituting (13) in (14), the probability will be given by

$$
\pi_{n}(2)=\int_{0}^{\frac{\left(\sigma_{1}+\sigma_{0}\right) \sqrt{\gamma_{L}}}{2 R P_{t}}} \frac{1}{\sqrt{8 \pi} h \sigma_{x}} \exp \left(-\frac{\left[\ln (h)+2 \sigma_{x}^{2}\right]^{2}}{8 \sigma_{x}^{2}}\right) d h
$$

Note that $\pi_{n}(2)$ can be considered as the cumulative distribution function (CDF) of the lognormal fading. Therefore, if we call this function $c d f_{I}(x)$, the transition probability will be

$$
\pi_{n}(2)=c d f_{I}\left(\frac{\left(\sigma_{1}+\sigma_{0}\right) \sqrt{\gamma_{L}}}{2 R P_{t}}\right)
$$

It can be shown that integration in Eq. (15) can be calculated as

$$
\pi_{n}(2)=\frac{1}{2}+\frac{1}{2} \operatorname{erf}\left[\frac{1}{\sqrt{8} \sigma_{x}}\left(\ln \left(\frac{\left(\sigma_{1}+\sigma_{0}\right) \sqrt{\gamma_{L}}}{2 R P_{t}}\right)+2 \sigma_{x}^{2}\right)\right]
$$

or in terms of SNR, it is expressed by

$$
\pi_{n}(2)=\frac{1}{2}+\frac{1}{2} \operatorname{erf}\left[\frac{1}{\sqrt{8} \sigma_{x}}\left(\ln \left(\sqrt{\frac{\gamma_{L}}{\bar{\gamma}}}\right)+2 \sigma_{x}^{2}\right)\right]
$$

This probability is numerically shown in Figs. 3 and 4 for different values of transmitted power and $\sigma_{x}$, representing fading strength. The threshold SNR $\gamma_{L}$ is chosen at a value of $4 \mathrm{~dB}$ that provides an extremely low probability of reconfiguration.

Similarly the probability of being at state 0 at time $n$, $\pi_{n}(0)$, can be calculated and expressed by 


$$
\pi_{n}(0)=\int_{\frac{\left(\sigma_{1}+\sigma_{0}\right) \sqrt{\gamma_{H}}}{2 R P_{t}}}^{\infty} f_{x}(h) d h
$$

It is clear that Eq. (19) will be calculated by

$$
\pi_{n}(0)=\frac{1}{2}-\frac{1}{2} \operatorname{erf}\left[\frac{1}{\sqrt{8} \sigma_{x}}\left(\ln \left(\frac{\left(\sigma_{1}+\sigma_{0}\right) \sqrt{\gamma_{H}}}{2 R P_{t}}\right)+2 \sigma_{x}^{2}\right)\right]
$$

In terms of SNR, it is expressed by

$$
\pi_{n}(0)=\frac{1}{2}-\frac{1}{2} \operatorname{erf}\left[\frac{1}{\sqrt{8} \sigma_{x}}\left(\ln \left(\sqrt{\frac{\gamma_{H}}{\bar{\gamma}}}\right)+2 \sigma_{x}^{2}\right)\right]
$$

Finally, the probability of being at state 1 as hysteresis gap can easily be calculated by

$$
\pi_{n}(1)=1-\pi_{n}(0)-\pi_{n}(2)
$$

where $\pi_{n}(0)$ and $\pi_{n}(2)$ are given by Eqs. (20) and (21), respectively. It can be easily realized that the reconfiguration probability is equal to:

$$
p_{12}=\pi_{n}(2)
$$

and the probability of recovering from an obscured link, the switch back probability, is given by

$$
p_{10}=\pi_{n}(0)
$$

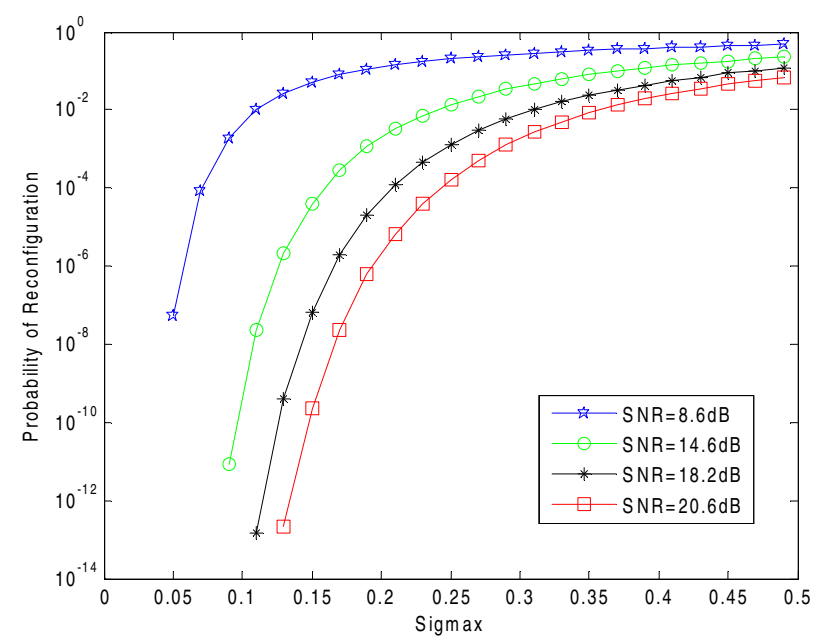

Fig. 3: Probability of reconfiguration, $p_{12}$, vs. fading for lognormal channel at different values of averaged SNR. $\gamma_{L}=4 \mathrm{~dB}$.

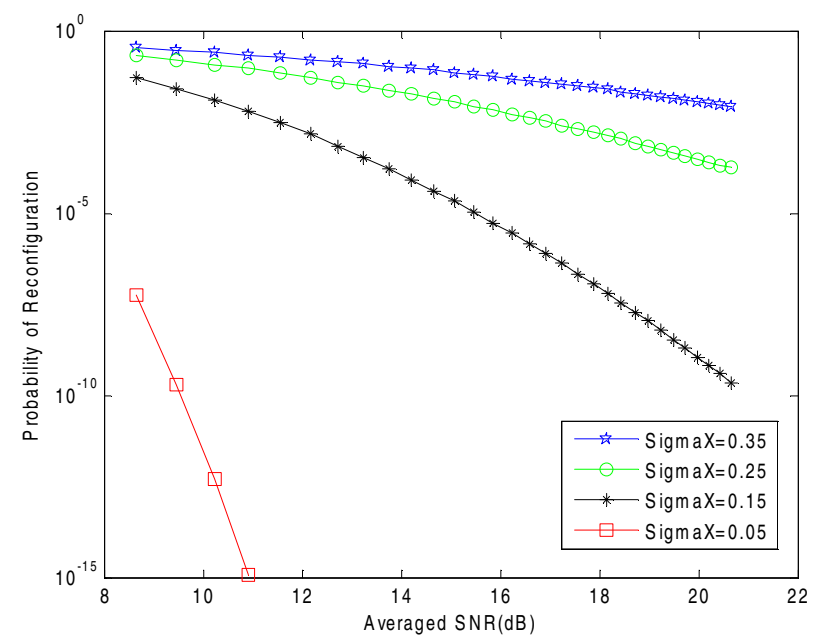

Fig. 4: Probability of reconfiguration, $p_{12}$, vs. averaged SNR for lognormal channel at different values of $\sigma_{x} \cdot \gamma_{L}=4 \mathrm{~dB}$.

\section{B. Finding Critical Cases}

If we want to find the critical points of $p_{12}$, i.e. $\pi_{n}(2)$, versus $\sigma_{x}$, it is adequate to apply Fermat's theorem. As shown in the Appendix, there is a local minimum point for the probability, which is

$$
\hat{\sigma}_{x}^{2}=\frac{1}{2} \ln \left(\frac{\left(\sigma_{1}+\sigma_{0}\right) \sqrt{\gamma_{L}}}{2 R P_{t}}\right)
$$

or,

$$
P_{t}=\frac{\left(\sigma_{1}+\sigma_{0}\right) \sqrt{\gamma_{L}}}{2 R} e^{-2 \hat{\sigma}_{x}^{2}}
$$

Since $\ln (x)<0$ for $x<1$, equation (25) cannot be valid as an extremum point for probability unless

$$
P_{t}<\frac{\left(\sigma_{1}+\sigma_{0}\right) \sqrt{\gamma_{L}}}{2 R}
$$

which represents low values of the transmitted power (i.e. small SNRs). Applying Eq. (4) can express the criteria expressed in terms of signal to noise ratios:

$$
\bar{\gamma}<\gamma_{L}
$$

When using this criteria and evaluating the second derivative test of $\pi_{n}(2)$ from the Appendix as

$$
\left.\frac{\partial^{2} \pi_{n}(2)}{\partial \sigma_{x}^{2}}\right|_{\hat{\sigma}_{x}}=\frac{1}{\sqrt{8 \pi} \hat{\sigma}_{x}^{3}} e^{-y^{2}} \ln \left(\frac{\gamma_{L}}{\bar{\gamma}}\right),
$$

is always positive for any $\bar{\gamma}$ satisfying (28). We can conclude that the reconfiguration probability has a local minimum point for $\pi_{n}(2)$, as is shown in Fig. 5. This indicates that increasing the fading strength of the channel does not always degrade link performance of FSO in terms of reconfiguration. The possibility of having a minimum point depends on satisfying the condition (27). However, the local minimum for reconfiguration probability appears in a regime where reconfiguration probability is high compared to Fig. 3. The threshold SNR $\gamma_{L}$ is chosen as $15 \mathrm{~dB}$, which is relatively high in wireless communication. Fig. 6 shows the probability for a different value of $\gamma_{L}=12 \mathrm{~dB}$. In this case, there is no local minimum point for probability reconfiguration at $\mathrm{SNR}=12.7$. However, if the system works at a lower $\bar{\gamma}$, e.g., $10.2,6.7$ or $0.66 \mathrm{~dB}$, local minimum points will exist.

\section{CONCLUSIONS}

An analytical investigation for characterizing reconfiguration probabilities in hybrid FSO/RF networks was conducted. Reconfiguration denotes the case that the traffic on a given FSO links should be directed to a different FSO link or even an RF link as backup. We applied a turbulence induced fading on the FSO link to model the performance fluctuation. The FSMC model has been used for probability analysis through reconfiguration occurrence in view of averaged received SNR. The authors stochastically derived closed-form expressions, describing the reconfiguration process through stationary and transition probabilities. It was demonstrated that increasing the intensity of lognormal fading does not necessarily increase the reconfiguration probability. Considering the interconnection application of an FSO communication network, the results can 
be applied for modeling and analyzing the rerouting and availability probabilities in such systems.

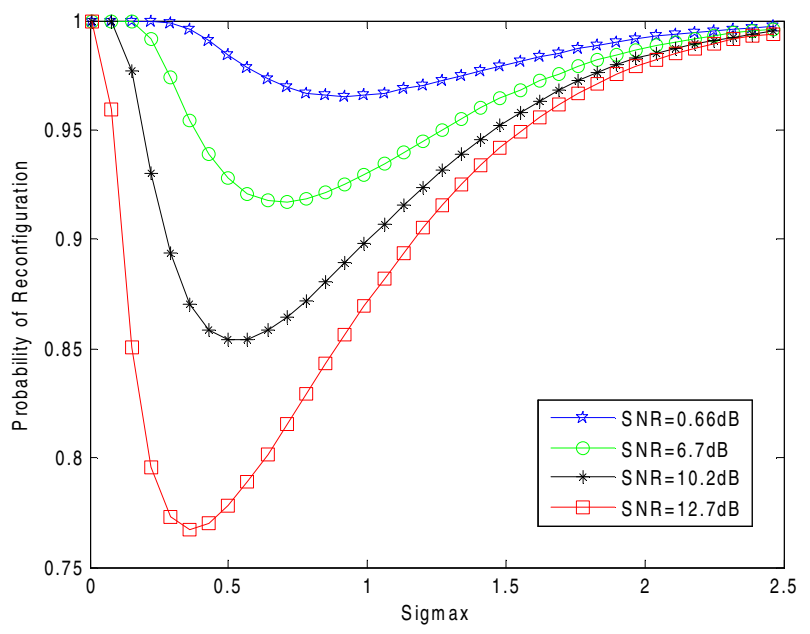

Fig. 5: Probability of reconfiguration, $p_{12}$, vs. fading for lognormal channel at different values of averaged SNR. $\gamma_{L}=15 \mathrm{~dB}$.

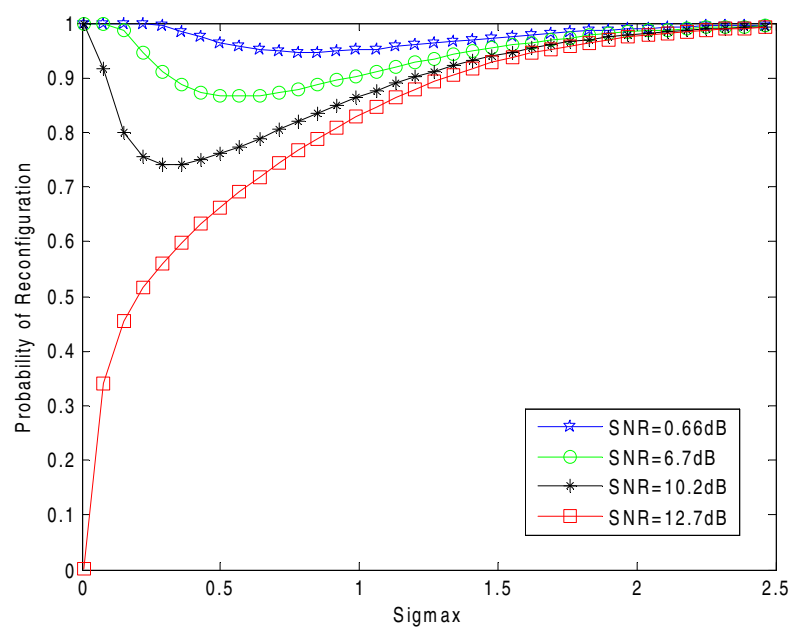

Fig. 6: Probability of reconfiguration, $p_{12}$, vs. fading for lognormal channel at different values of averaged SNR. $\gamma_{L}=12 \mathrm{~dB}$.

\section{ACKNOWLEDGMENTS}

This work is funded by NSF grant number NSF-ECCS 0725801 .

\section{APPENDIX}

Proof of (25) and (29): The derivative of Eq. (20) will be given by

$$
\frac{\partial \pi_{n}(2)}{\partial \sigma_{x}}=-\frac{1}{2} \frac{\partial \operatorname{erf}(y)}{\partial \sigma_{x}}=-\frac{1}{2} \frac{\partial \operatorname{erf}(y)}{\partial y} \frac{\partial y}{\partial \sigma_{x}}
$$

in which $y=\frac{1}{\sqrt{8} \sigma_{x}}\left(\ln \left(\frac{\left(\sigma_{1}+\sigma_{0}\right) \sqrt{\gamma_{L}}}{2 R P_{t}}\right)+2 \sigma_{x}^{2}\right)$. Since

$\frac{d}{d x} \operatorname{erf}(x)=e^{-x^{2}}$, the differential equation in question is calculated by

$$
\frac{\partial \pi_{n}(2)}{\partial \sigma_{x}}=-\frac{1}{2} \frac{2}{\sqrt{\pi}} \frac{1}{\sqrt{8}} e^{-y^{2}}\left(2-\frac{\ln \left(\left(\sigma_{1}+\sigma_{0}\right) \sqrt{\gamma_{L}} / 2 R P_{t}\right)}{\sigma_{x}^{2}}\right)
$$

By considering $\frac{\partial \pi_{n}(2)}{\partial \sigma_{x}}=0$, equation (25) is easily achieved. Also, the second order derivative of $\pi_{n}(2)$ versus $\sigma_{x}$ is expressed by

$$
\begin{aligned}
& \frac{\partial^{2} \pi_{n}(2)}{\partial \sigma_{x}^{2}}=\frac{1}{\sqrt{2 \pi}} e^{-y^{2}} \\
& \quad \times\left(\frac{\ln \left(h_{L}\right)}{\sigma_{x}^{3}}+y\left(-\frac{1}{\sqrt{8} \sigma_{x}^{2}} \ln \left(h_{L}\right)+\frac{\sqrt{2}}{2}\right)\left(2-\frac{\ln \left(h_{L}\right)}{\sigma_{x}^{2}}\right)\right)
\end{aligned}
$$

where $h_{L}=\left(\sigma_{1}+\sigma_{0}\right) \sqrt{\gamma_{L}} / 2 R P_{t}$. By substituting (25) in (32) for $\sigma_{x}$, (29) will be achieved.

\section{REFERENCES}

[1] Gurumani, S., Moradi, H., Refai, H. H., LoPresti, P. G., Atiquzzaman, M., Dynamic Path Reconfiguration among Hybrid FSO/RF Nodes, IEEE GLOBECOM'08, pp. 1-5, Nov. 2008.

[2] Wang H., S., Moayeri, N., Finite-state Markov channel-a useful model for radio communications channels, IEEE Trans. on Vehicular Tech., Vol. 44, No. 1, pp. 163-171, 1995.

[3] Gilbert, E.N., Capacity of a burst-noise channel, Bell Syst. Tech. J., vol. 39, no. 9, pp. 1253-1265, 1960.

[4] Elliott, E.O., Estimates of error rates for codes on burst-noise channels, Bell Syst. Tech. J., vol. 42, no. 5, pp. 1977-1997, 1963.

[5] Arauz, J., Krishnamurthy, P., and Labrador, M. A., Discrete Rayleigh fading channel modeling, Wiley Journal of Wireless Comm. and Mobile Computing, pp. 413-425, Wiley \& Sons Inc., 2001.

[6] Wilfert, O., and Kolka, Z., Statistical Model of Free-Space Optical Data Link, Proceedings of the SPIE'04, Vol. 5550, pp. 203-213, Aug. 2004.

[7] Kim, I. I., and Korevaar, E., Availability of Free Space Optics (FSO) and Hybrid FSO/RF Systems, Proceedings of SPIE, Vol. 4530, pp. 84-95, Aug. 2001.

[8] Wang, H. S., and Chang, P. C., On verifying the first-order Markovian assumption for a Rayleigh fading channel model, IEEE Trans. Veh. Tech., vol. 45, pp. 353-357, May 1996.

[9] Hajjarian, Z., Fadlullah, J., Kavehrad, M., Use of Markov chain in atmospheric channel modeling of free space laser communications, IEEE Milcom'08, pp. 1-7, 2008.

[10] Moradi, H., Falahpour, M., Refai, H. H., LoPresti, P. G., Atiquzzaman, M., Toward an Optimal Combined FSO/RF System via an Adaptive Bit Rate Control , SPIE Photonic West, 2010.

[11] Sadeghi, P., Kennedy, R. A., Rapajic, P. B., and Shams, R., Finite-state Markov modeling of fading channels - a survey of principles and applications, IEEE Signal Processing Magazine, Volume 25, Issue 5, pp. 57-80, 2008.

[12] Hoel, P. G., Port, S. C., Stone, C. J., Introduction to Stochastic Processes, Waveland Press Inc., 1987.

[13] Navidpour, S. M., Uysal, M., and Kavehrad, M., BER Performance of FreeSpace Optical Transmission with Spatial Diversity, IEEE Trans. Wireless Comm., Vol. 6, No. 8, pp. 2813-2819, Aug. 2007. 